\title{
A Study on the Control and Guidance Strategy of the Diaolou Style Building of Qiang People Based on Behavioral Analysis
}

\author{
Bin Cheng, Libin Duan, Haifeng Lan \\ Southwest University of Science and Technology, Mianyang, China \\ Email: chengbin1971@126.com
}

How to cite this paper: Cheng, B., Duan, L.B. and Lan, H.F. (2018) A Study on the Control and Guidance Strategy of the Diaolou Style Building of Qiang People Based on Behavioral Analysis. Journal of Building Construction and Planning Research, 6, 162-184. https://doi.org/10.4236/jbcpr.2018.63012

Received: July 13, 2018

Accepted: September 26, 2018

Published: September 29, 2018

Copyright ( 92018 by authors and Scientific Research Publishing Inc. This work is licensed under the Creative Commons Attribution International License (CC BY 4.0). http://creativecommons.org/licenses/by/4.0/

\begin{abstract}
Diaolou style building is an important residential building of Qiang people. In recent years, Qiang people's own cultural identities and behavioral awareness have transformed. Due to the lack of attention to the inheritance of original architectural style and the absence of a detailed scientific protection of the landscape, the architectural style of Diaolou style building gradually loses its vitality. It is difficult to control and guide it effectively. And tourism economy which enjoys a direct link with the architecture has been confined to further development. From the perspective of behavioral science, this study innovatively analyzes the Diaolou style building of Qiang people by combining the methodology of Lew in metal of behavior and Analytic Hierarchy Process. Firstly, 32 symbols are chosen as Diaolou architectural style factors from the selection of more than 100 Diaolou architectural decorative symbols. Then by measuring their weight coefficients through the Analytic Hierarchy Process, eight main style factors have been screened out. After field research, we analyze the actual use of the style factors of the six typical villages such as Gina. On this basis, this paper analyzes the behavioral factors of Diaolou style building of Qiang people, deconstructs its use and puts forward a concept which takes style control as a guidance and behavior guidance as a strategy to protect and develop Diaolou style building of Qiang people. It is also proposed that a control and guidance strategy of inheritance and development of Qiang Diaolou architectural style, including master the influencing factors as a whole, follow the principle of people-oriented, compound function, dynamic development and highlighting national aesthetics, based on its style system, and grasp the trend of architectural style, etc. The study provides an effective guarantee for the further development of rural tourism, and a necessary theoretical and technical guidance for the government to adopt targeted steps.
\end{abstract}




\section{Keywords}

Diaolou Style Building, Style Factor, Behavioral Factors, Diaolou

Architecture, Qiang People, Cultural Identity

\section{Introduction}

\subsection{Research Background}

Diaolou style building of Qiang people is not only the most important residential building, but also an important carrier of Qiang culture in Qiang fortress villages. It plays an irreplaceable role in shaping the regional culture of Qiang nationality and displaying the traditional art and national architecture. It is the inevitable requirement for maintaining and developing buildings of Qiang nationality to study the characteristics of Diaolou style building of Qiang people and analyze their protective strategies.

\subsection{Research Progress}

\subsubsection{Literature Review}

In the academic field, there are some related researches on Diaolou style building of Qiang people. Ma [1] applied to the theory of folklore to analyze the construction, shape, scale, category and function of Qiang Diaolou, which revealed a deep-seated national culture connotation and evaluated the value of Qiang people's Diaolou. Li [2] introspected the design of the external space of modern architecture by analyzing the interface and contents of Diaolou style building of Qiang people. Yu [3] analyzed the ecological design of Diaolou from the aspects of site selection, space combination, building material, structure treatment, etc., and revealed the original ecological concepts of traditional Chinese vernacular housing. Yong [4] analyzed the superior seismic performance of Diaolou from its site selection, timber, structural arrangement and construction quality. And he expounded Qiang architectural culture from the architectural structure. Zhang [5] employed anthropology and architecture theory to analyze the distribution, construction, function, category and scale of Diaolou of Qiang ethnic group, which exposed the architectural features and cultural connotations implied by Diaolou, and further explored the value and protection of Diaolou. Professor Ji Fuzheng [6] [7] who is from Southwest Jiaotong University, has conducted a systematic research and comprehensive mapping of Qiang dwellings. He has explored the initial state of houses in the Three Gorges Area based on archaeological data and ancient cultural sites in the Three Gorges area, and has analyzed the origins and changes of housing settlements in the area. Gan [8] analyzed the spatial landscape and the building space of Qiang people. Peng [9] analyzed the art form and functional characteristics of this architecture, then healso analyzed the functions of family identity and religious worship. After Wenchuan earthquake, under the support of reconstruction policy, the types of 
residential and public buildings are more diversified and richer in forms. The cultural connotation of the Qiang people's dwellings continues to rise, reflecting era characteristics. Yang [10] conducted a study on the architectural restoration of Wenchuan from architectural design to project implementation. Wan [11] analyzed the characteristics of Qiang buildings from aspects of earthquake resistance, defensiveness and energy conservation, and studied the practical value on the basis of conservation value. Ren [12] analyzed materials and technology of the Qiang ethnic building. Wu [13] studied the Baishi symbol and its cultural characteristics in Qiang buildings. In the reconstruction of modern culture and national culture, the Qiang architectural restoration and renewal have been studied [14]. Liu [15] mainly focused on the architectural style of Zhuang Fang, analyzed its architectural structure, environmental art, and cultural beliefs that affected the distribution of architecture. Yuan [16] conducted a study on the site selections, layouts, architectural types and features of ancient Qiangzhai. At the same time, Many researchers [17] [18] [19] [20] made an in-depth study of the evolvement direction of traditional Qiang buildings and put forward that it was necessary to develop residential areas in the new era, to adapt to the needs of modern life, and to return to the national tradition on the basis of keeping the tradition.

Through the analysis of the existent research, the following conclusions can be drawn. First, the majority of studies of Qiang buildings and Diaolou style architecture are concerned with architectural forms and local decorative features. Therefore, there is a lack of systematic study on the style factor system of Diaolou architecture. Second, there is a lack of research on the style protection of Qiang buildings from the perspective of behavioral control strategy. As a result, in spite of related achievements in the researches on the architectural features of Qiang, they have not been fully utilized in the practice of Qiang construction in recent years.

\subsubsection{Questions Raised}

Based on the analysis of the research and the actual investigation, it is found that even in the typical Qiangzhai, the unique ethnic style, geographical and cultural feelings of Diaolou style building of Qiang people have not been fully demonstrated; the features of the regional landscape are gradually lost, and the unique architecture culture is gradually extinguished.

Specific reasons are as follows: first, due to the weakening of their own cultural identity, the original elements of Diaolou style building have not been taken seriously, resulting in the gradual loss of architectural features. Second, the understanding of the usage behavior of the style factor in Diaolou style building of Qiang people is vague, thus, it is difficult to guide it by science. As a result, the style factor of Diaolou style building has a large amount of heterogeneity in use. Third, the development of modern architectural technology has impacted the original Qiang's construction techniques, resulting in the abandonment of the original features. Fourth, there is a lack of interpretation of the Qiang people's 
architectural style and cultural system, so a complete Qiang architectural culture system has not yet been formed, resulting in a separation of the new building from the existing cultural environment.

\subsubsection{Countermeasures}

The key to solve the above problems is to study and analyze the Diaolou style building of Qiang people system and to deconstruct the use of style factor in Diaolou style building. The reasons are as follows: firstly, improve the Qiang cultural system through the construction of Diaolou architectural style system. By excavating the unique value of the Diaolou style building culture system, it is conducive to regaining national confidence and attaching importance to the protection of the style. Secondly, through the analysis of behavioral factor, the actual use of style factor in Diaolou style building is oriented, and the behavior pattern is deconstructed; analyze the behavioral factor that inhibits or promotes the display of architectural style in Diaolou style building of Qiang people, so as to promote the conduct of behavioral control and guidance strategy. Thirdly, by deconstructing factor system in Diaolou style building of Qiang people, the backward relationship between the style factors in Diaolou style building and the existing construction techniques is analyzed. Through the use of Qiang building system, modern people can improve the building techniques, and modern technology can be adapted to the development of the nation. Fourthly, it is helpful to recognize the trend of development of Qiang building system and the current cultural system to adapt the architectural form to the social development.

\subsection{Research Content}

\subsubsection{Research Priorities}

On the basis of comprehensive analysis, this study adopts the method of combining Analytic Hierarchy Process and Lewin metal of behavior, focusing on the style system of Diaolou style building of Qiang people and deconstructing the use of style factors of Diaolou style building. It analyzes the use of style factor system in the Diaolou style building of Qiang people from the behavioral perspective, and proposes a scientific behavior control and guidance strategy for the inheritance and development of the Qiang Diaolou architectural style. It provides the necessary technical theory and landscape renovation thought for the governments and planners' practical work.

\subsubsection{Research Highlights}

The research perspectives and methods of this paper are innovative. In terms of research perspectives, this paper studied the use of style factor in Diaolou style building of Qiang people from the perspective of behavior pattern. In terms of research methods, the subjective style and behavior problems were quantified in combination with the survey, making the research more scientific and the survey results more intuitive. In addition, the control and guidance strategy have been proposed, which provide not only an effective guarantee for the further devel- 
opment of rural tourism, but also a necessary theoretical and technical guidance for the government to adopt targeted steps.

\section{Method}

\subsection{Selection of Research Methods}

\subsubsection{Analytic Hierarchy Process}

Style factor system of Diaolou style building of Qiang people is an intertwined system, involving the factors of volumetric form and architectural decoration. The existing theory about the construction of Diaolou style building of Qiang people is still based on principles of architectural design and classifications of types, but it lacks a quantitative analysis of the architectural style factor system. At the same time, the existing building index system cannot fully describe the architectural features of Diaolou style building of Qiang people. The reasons are as follows: first, the traditional architectural index system has its own inherent disadvantages. The architectural style is mainly characterized by general architectural features, but it is in want of the accurate alignment and the quantization of importance. Second, the traditional architectural index system cannot take into account national characteristics and geographical differences, so that cultural focus is not allowed. Thus, it cannot be fitted with the unique architecture in Qiang region. Third, the static traditional building index system fails to make predictions from the perspective of the evolvement in actual usage behavior, and to adapt to the ever-changing architectural features of the landscape. To sum up, therefore, it is necessary to establish an accurate and refined style factor system of Diaolou style building of Qiang people that not only can achieve clearly defined primary and secondary, but can adapt to development and transformation.

The Analytic Hierarchy Process was proposed by the American operational research expert, Thomas L. Saaty [21] [22] from University of Pittsburgh for complex decisions in the early 1970s. According to the subjective judgment of a certain objective reality, users of the AHP first decompose their decision problem into a hierarchy of more easily comprehended sub-problems. Then the decision makers evaluate its various elements by comparing them to each other two at a time, with respect to their impact on an element above them in the hierarchy by combining the experts' opinions with the analysts' objective judgments directly. Because of the need to quantify and analyze the importance of style factor system of Diaolou style building of Qiang people, and there is a high degree of similarity between style factor system of Diaolou style building and AHP which can be employed to describe problems in complex situations. Therefore, this study uses AHP to analyze style factor system of Diaolou style building.

\subsubsection{Lewin Metal of Behavior}

Lewin Metal of Behavior [23] mainly studies the interrelationship between internal and extrinsic factors, and defines behavior as a function of the person in the environment. He proposes the Behavioral Model Theory that $\mathrm{B}=f(\mathrm{P}, \mathrm{E})$. 


$$
B=f(P, E)
$$

where $B$ is behavior, $P$ is person, and $E$ is the environment.

Lewin Metal of Behavior states that behavior is a function of the person in their environment, which is consistent with the behavior of style factors of Diaolou style building in this study. The use of styles factor in Diaolou style building involves three aspects: the user, the environment and the characteristics of the factor. Therefore, the Lewin Metal of Behavior is adjusted to suit for the research.

On this basis, this study proposes a behavioral model that $B=f(P, E, M)$, which is based on the interaction of three factors: person, environment and object of selection.

$$
B=f(P, E, M)
$$

where $B$ represents behavior, $P$ isperson, $E$ is environment, and $M$ is selected object.

After analyzing the Lewin Metal of Behavior, we can draw a conclusion that the final choice of behavior is determined by person, object and environment. In order to quantitatively describe the effect of different impact factors, this study continues to use AHP.

The reason is as follows: first, the usage behavior involves the interweaving of the multiple choice behaviors. Second, the importance of the decision of the choice behavior needs to be quantitatively described. Third, it involves the comparison of different factors, so it continues to use the Analytic Hierarchy Process to quantitative analysis.

\subsection{Research Model}

Based on the qualitative and quantitative analysis, research about guidance strategy of behavior control in Diaolou style building of Qiang people combines Analytic Hierarchy Process with Lewin Metal of Behavior.

First of all, Analytic Hierarchy Process is used to screen the style factor of Qiang folk housing to establish a factor system of Diaolou style building of Qiang people.

Second, the Lewin Metal of Behavior is used to analyze the behavior of main style factors and to derive the behavioral factors that influence the use of the style factors.

Third, use the Analytic Hierarchy Process to analyze the main behavioral factors.

According to the research model (see Figure 1), the following research steps are established:

1) Establishing a style factor system of Diaolou style building of Qiang people;

2) Analyzing style factor system in Diaolou style building of Qiang people hierarchically;

${ }^{1}$ https://baike.baidu.com/item/\%E5\%8D\%A2\%E5\%9B\%A0\%E8\%A1\%8C\%E4\%B8\%BA\%E6\%A8\%A $1 \% \mathrm{E} 5 \% 9 \mathrm{E} \% 8 \mathrm{~B} / 2362899$ ?fr=Aladdin.

${ }^{2}$ According to formula 1 , the author himself modified the formula. 


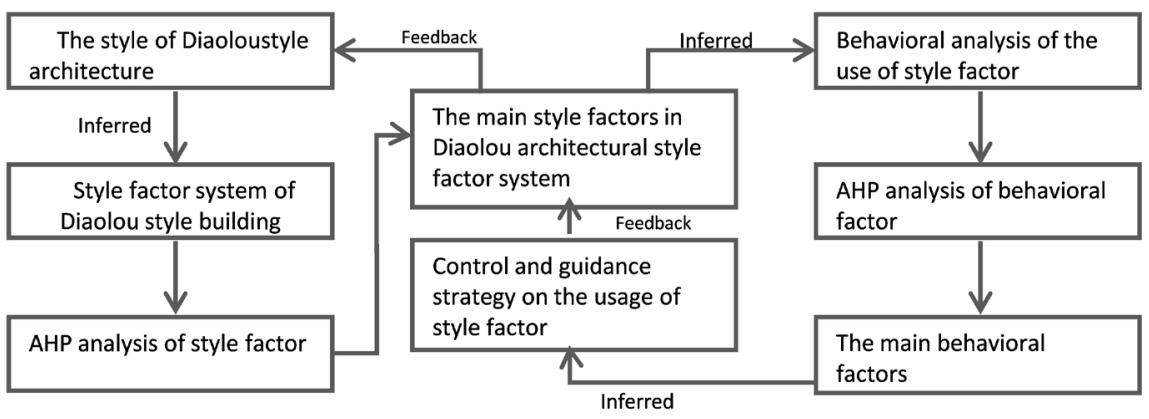

Figure 1. Study model of control and guidance strategy on diaolou style architecture.

3) Obtaining the weight value of the main style factors in Diaolou architectural style factor system;

4) Establishing impact factors of each usage behavior in the system for each main style factor;

5) Analyzing the impact factors of each usage behavior impact factors in each usage behavior impact factors system of each main style factor;

6) Obtaining the weight value of each usage behavior impact factors in each usage behavior impact factors of each main style factor system;

7) Corresponding to the weight value of each usage behavior impact factor in the behavior impact factor system, and weighting according to the weights of the main style factors in the Diaolou architectural style factor system;

8) Obtaining main impact factors of usage behavior of Diaolou architectural style factor.

\subsection{Experiment}

\subsubsection{Study Area Selection (Figure 2)}

Aba is located in the northwestern part of Sichuan Province, China, which lies in the southeastern margin of the Qinghai-Tibet Plateau, the junction of the northern end of the Hengduan Mountains and the alpine valley in the northwest of Sichuan. The landforms are mainly high plateau and high mountain canyon, the southeast is the high mountain canyon area, the middle is the mountain area, and the northwest is the plateau area. Wenchuan, Lixian and Maoxian of Aba Prefecture are the main settlements of Qiang people. In the Wenchuan earthquake, indigenous architectures were greatly damaged and under the invasion of foreign culture, these architectural styles have been disappearing. In order to truly reflect the style of Qiang indigenous architecture, this paper choosed 6 representative Qiangzhai (Qiang villages) as the research object.

\subsubsection{Qiang Diaolou Building Style System}

The research on the construction of Diaolou architectural style factor system of Qiang nationality is based on Jencks's [24] interpretation of the new paradigm of architecture. Through field investigation and analysis, the style factors of Diaolou style building have been summarized. After classifying style factors and analyzing the correlation between different factors, a relational model of Diaolou 


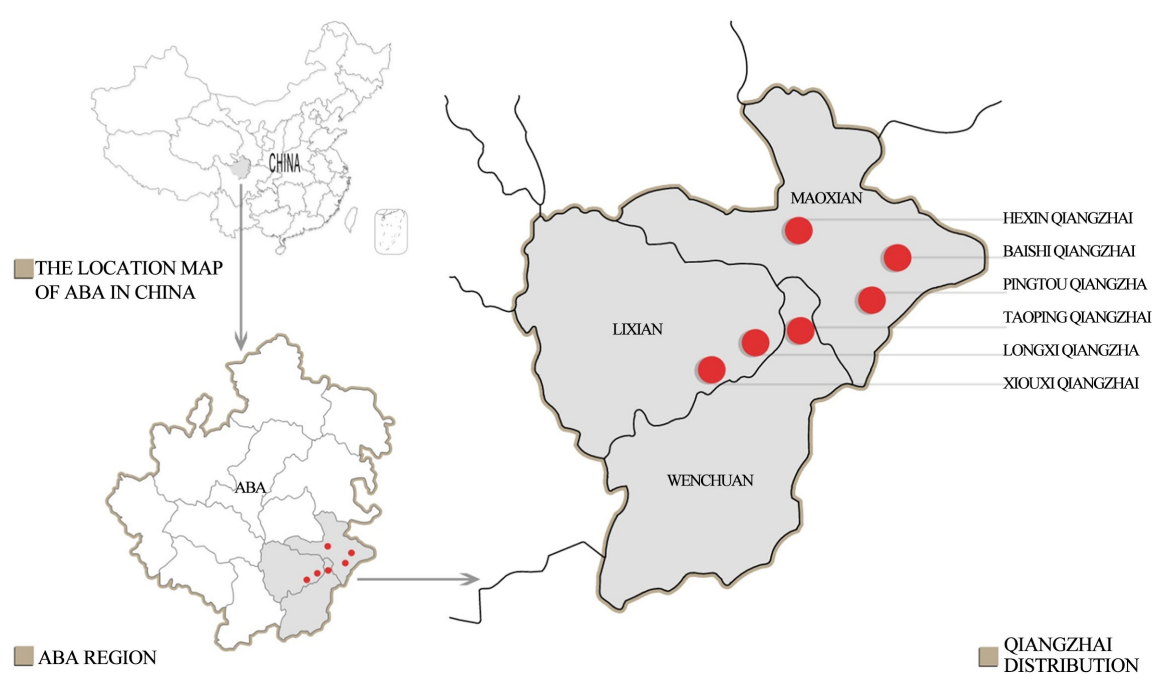

Figure 2. Study area distribution.

architectural style factors can be constructed. On this basis, the importance of style factors in Diaolou style building is quantified, and the characteristics of which are deconstructed.

1) Selection principle of style factors: according to the analysis of the urban image by Kevin Lynch [25] and the reference to three aspects of the social effect, ecological quality and aesthetic effect of landscape factors in the landscape evaluation theory of Xie [26]. Therefore, based on the principles of Yang [27], five principles of comprehensiveness, typicality, science, hierarchy and feasibility are finally adopted.

2) Selection of style factors: according to the selection principles of Che \& Yang [28] [29], four steps are applied to evaluate and determine the characteristics of the style factor.

a) Initial impression method: after accessing information and collecting literature, factors are settled.

b) Expert screening method: according to the selection principle of factors, invite five experts to conduct a preliminary screening of factors.

c) Field research method: combined with the questionnaire, make an adjustment of the representative and regional factors.

d) Expert assessment method: continuing to invite five experts to identify the rationality of the factors to determine the characteristics of style factors according to the survey and the questionnaire. See Table 1.

3) Classification of style factors: based on the principles of hierarchy and feasibility of the selection of factors, and the hierarchical classification theory of analytic elements in AHP, the style factors of Diaolou style building of Qiang people are divided into three levels which include 4 categories and 32 sub-categories (Figure 3).

The form factors of the architectural style factors of Diaolou style building are as follows: 
The typical Qiang style building is composed of Semi-masking roof, Overhang the balcony, Set-back model, Body contraction, Nase, etc. Through the partial relief of these building forms, the roofs are scattered in different levels and combined with the Diaolou to form a distinctive façade and roof line. Its typical types of body symbol and examples are shown in Figure 4;

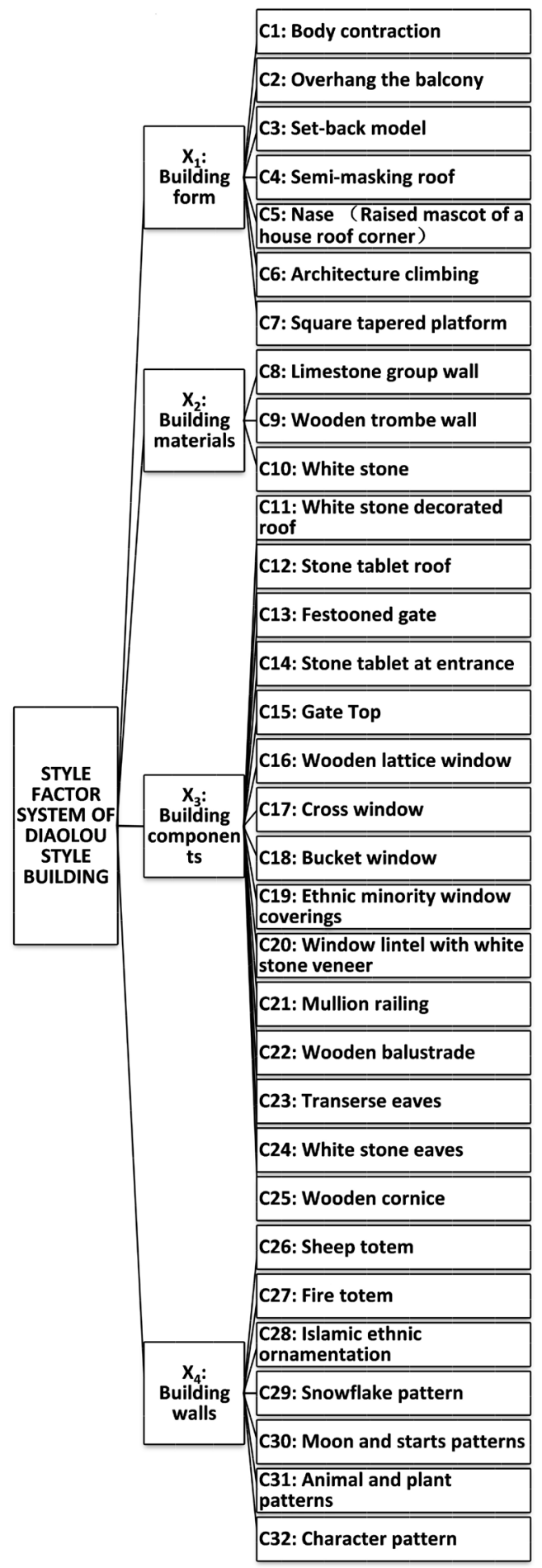

Figure 3. Style factor system of diaolou style building. 


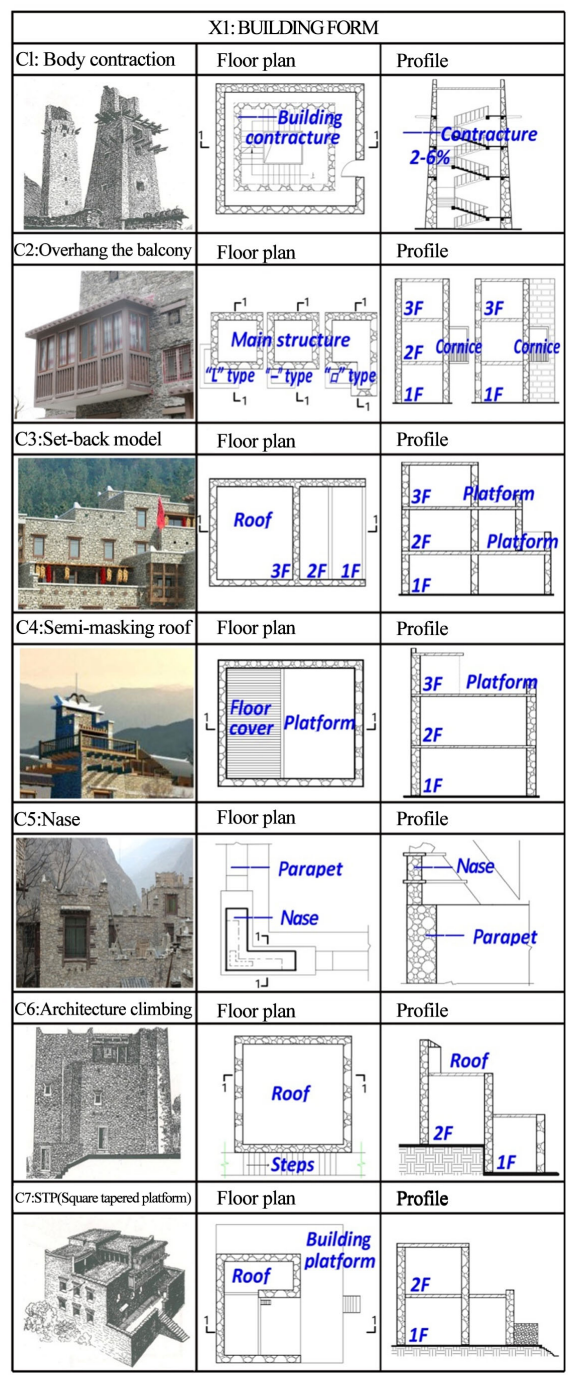

Figure 4. Factor diagram in the building form.

Table 1. Weight of Style Factor about Diaolou style building.

\begin{tabular}{cccc}
\hline factor & Weights & factor & Weights \\
\hline C1 & 0.0815 & C17 & 0.0069 \\
C2 & 0.0231 & C18 & 0.0081 \\
C3 & 0.0212 & C19 & 0.0501 \\
C4 & 0.0193 & C20 & 0.0072 \\
C5 & 0.0821 & C21 & 0.0076 \\
C6 & 0.0161 & C22 & 0.0117 \\
C7 & 0.0188 & C23 & 0.0085 \\
C8 & 0.1245 & C24 & 0.0843 \\
C9 & 0.0389 & C25 & 0.0075 \\
C10 & 0.0487 & C26 & 0.0765 \\
C11 & 0.0608 & C27 & 0.0377 \\
\hline
\end{tabular}


Continued

\begin{tabular}{llll}
\hline C12 & 0.0124 & C28 & 0.0187 \\
C13 & 0.0107 & C29 & 0.0166 \\
C14 & 0.0025 & C30 & 0.0103 \\
C15 & 0.007 & C31 & 0.014 \\
C16 & 0.054 & C32 & 0.0125 \\
\hline
\end{tabular}

Building material (Figure 5): brownish-yellow or blue-gray stone constitutes the main timber of the exterior wall, and the grayish-gray tile serves as the timber of the sloping roof. Rough appearance, hard, clear texture and stitching patchwork display the heavy stone and hard texture. The color of the material is the basic color of the building.

Building roof: White stones are the core of decorative materials for the roofs of the Qiang style buildings, reflecting the texture and color. It is common to put white stones and stone tablets on the roofs for decoration.

Building entrance: the combination of Qiang-style festooned gate and stone wall is very harmonious; local structures such as davits and petals inherit Han-style skills and usually have two columns to support them. Stone tablets at entrance as an important Qiang cultural symbol, can ward off evil spirits; there is a door frame of the entrance in Qiang-style building. Generally speaking, double -door is more than single-door.

Building window: windows are an important symbol of a national architecture; Qiang-style windows enjoy distinctive features, including wooden lattice windows, bucket windows, ethnic minority windows, etc. Wooden lattice windows are connected with a national style of windows-Chinese-style windows, adding patterns of ram's horns and beef ribs into window frames. Bucket windows are common in the stone walls, including cross windows, bucket windows and other forms. Ethnic minority windows are mainly made of sheep totems, being attached to the window walls as a national decorative component.

Building railing: Generally speaking, Qiang-style railings include mullion railing, wooden balustrade, and other railings that combine horizontal with vertical elements; the overall impression is relatively simple, occasionally with exquisite railings.

Building eaves: Decorative effects of canopies in Qiang-style buildings can be summarized as the following: first, patterns such as palindromes and triangles appear in a canonical form in the eaves; second, in some traditional Qiangzhai, a bow-like member, buckled on the eaves, arranged in a row, is not only a very beautiful decoration but can also protect the wall; third, wider than the wall, stone tablets are employed at the top of the gable walls, serving a cornice-like function, to protect the walls from the rain. The above typical building components and examples are shown in Figure 6.

Building walls (Figure 7): graphics in white, yellow or red Sheep totems, fire totems, Islamic ethnic ornamentations and other types are placed on the walls by 
splicing, inlaying, hanging and other ways to form a colorful splicing. In addition, there are such other colors as black which is formed by toasted wood.

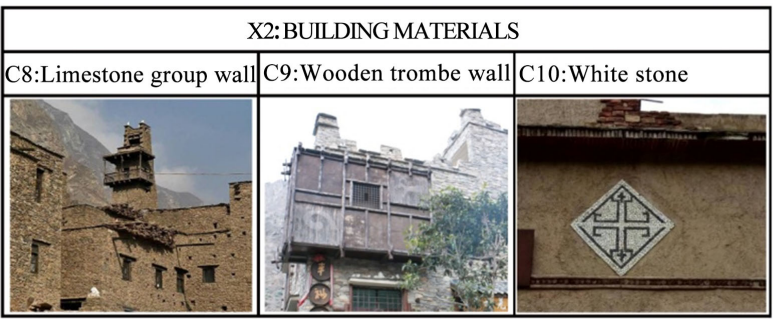

Figure 5. Factor diagram in building materials.

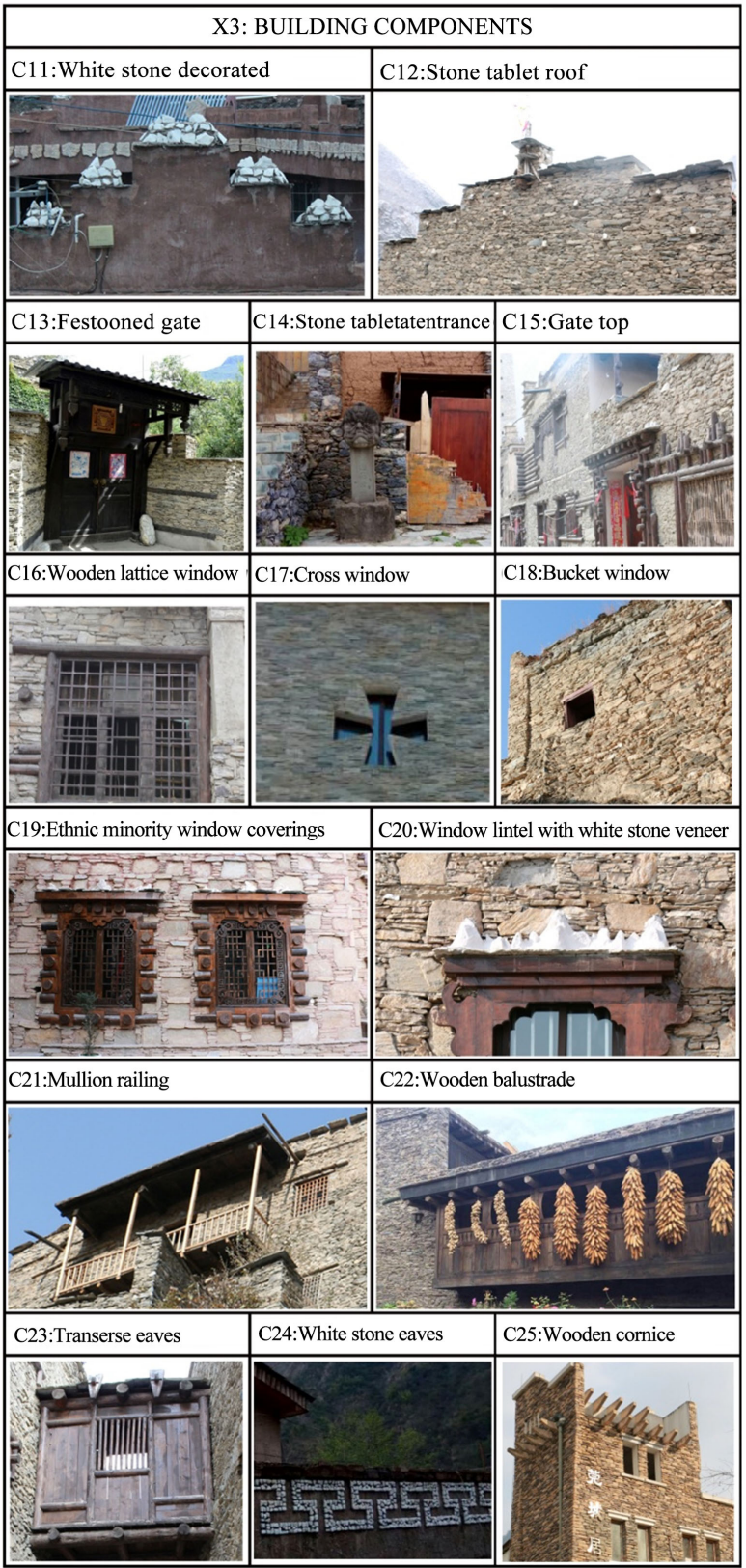

Figure 6. Factor diagram in building components. 


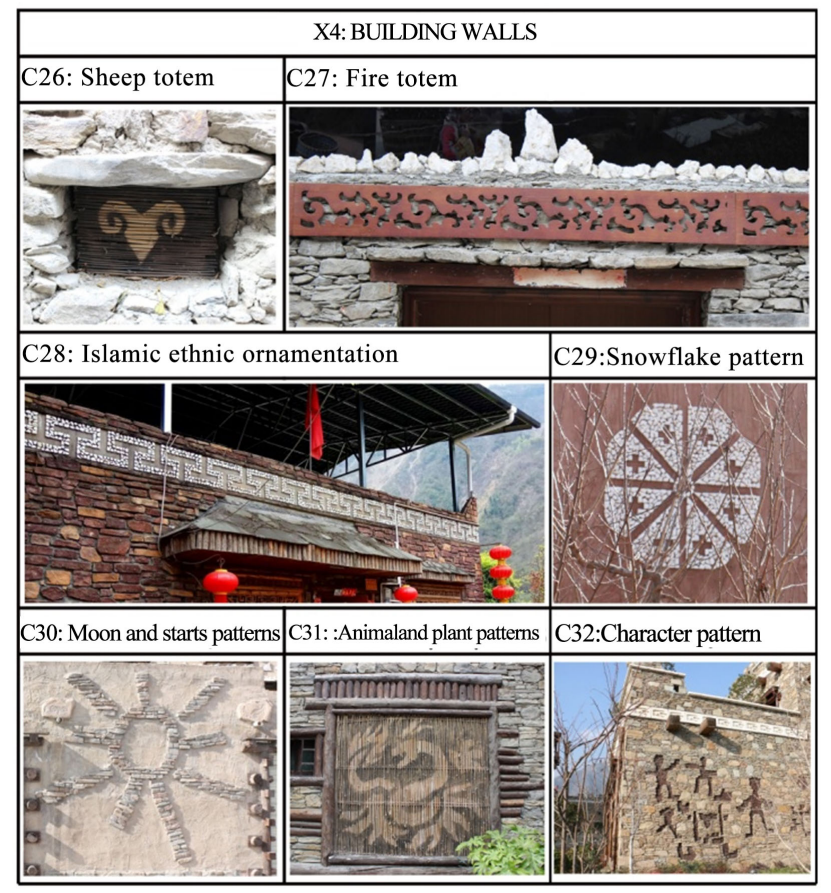

Figure 7. Diagram of factors in building walls.

\subsubsection{Analytic Hierarchy Process on Style System of Diaolou Style Building of Qiang people \\ According to Satty's AHP, methods of this study are as follows:}

1) Establishing the AHP model: based on the style factor system of Diaolou style building of Qiang people, problems that need to be analyzed are classified into three levels. The objective of the study is to highlight the style features of Diaolou style building of Qiang people. Four categories belong to the index of control level, while 32 sub-categories belong to the indicator of each level. Each control level affects its subordinate factors.

The AHP model of this study is constructed as follows (Figure 8):

2) Construction Judgment Matrix: Construct a judgment matrix to compare the importance of factors; consult such experts as senior designers in architectural design institute, personnel from university architecture academy, and architecture graduate students. The questionnaire adopts a nine-level evaluation system, and five experts (odd-numbered places) evaluate the importance of each index item and each intermediate factor.

3) Hierarchicalsingle sorting: normalize the judgment matrix and calculate the weight vector of the judgment matrix so as to obtain the weight value of the impact factors in the judgment matrix.

4) Consistency test: on the basis of weight vector, the maximum characteristic and the approximate value of the judgment matrix are obtained. According to the consistency of the Analytic Hierarchy Process, the consistency of the judgment matrix is verified.

5) Hierarchicaltotal sorting: according to the hierarchical model, turn judgment matrix into the hypermatrix and divide each weight vector in the 
judgment matrix. Then weight value of each factor in the level to a factor in the upper level of the judgment matrix is obtained; finally, using the method of weighted sum, the final weight of each indicator to the total goal is combined.

6) Result of weight: use Super Decisions software to calculate the following weight division (As shown in Table 1):

According to the weight of corresponding factor, results are as follows (As shown in Figure 9):

\subsubsection{Screening of Main Style Factor}

Through the screening of data, the style factors of Diaolou style building are divided into three categories: $\geq 0.05,0.02-0.05$ and $\leq 0.02$, which are respectively defined as the main, the secondary and the general style factors. Among them, the main style factors of Diaolou style building include limestone group wall, wooden lattice window, white decorated stone, white stone eave, window covering with ethnic characteristics, nase, body contraction and sheep totem; they

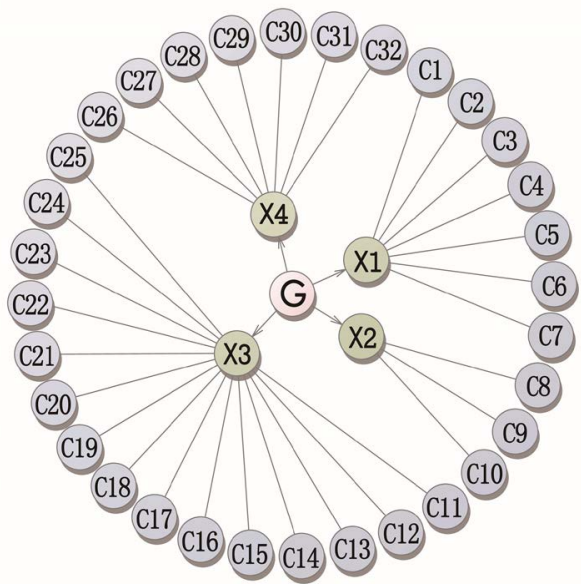

Figure 8. Analytical model of AHP.

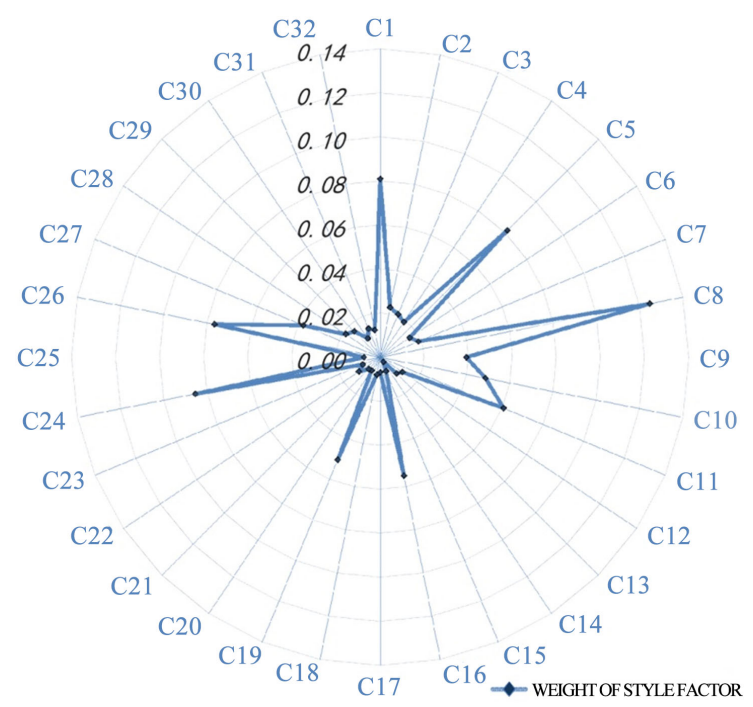

Figure 9. Weight of Style Factor aboutDiaolou style building. 
should be the focus of controlling and guiding construction activities for such factors occupy a large proportion of the architecture.

It can be seen from Figure 9 that the importance of style factors of Diaolou style building varies from factor to factor. To further describe the style of Diaolou style building, its factors and the actual use of the behavioral factor need a further analysis.

\subsubsection{Usage Behavior Impact Factors System of Style Factorin Diaolou}

Based on the Lewin Metal of Behavior, this study proposes a behavioral model of $B=f(P, E, M)$, which is a combination of three factors: person, environment and choice of object.

$$
B=f(P, E, M)
$$

where $B$ is behavior, $P$ is person, and $E$ is the environment. According to this model, the following set of behavior selection factors is established:

$\mathrm{Bi}=\{$ Limestone group wall, Wooden lattice window, White stone decorated roof, White stone eave, Ethnic minority window covering, Nase, Body contraction, Sheep totem

$\mathrm{P}=\{$ Aesthetic cognition, Income Level, Cultural Identity\}

$\mathrm{E}=\{$ Family influence, Cultural influence, Environment influence, Market supply\}

$\mathrm{M}=\{$ Innovation, Practicability, Durability, Safety, Price level $\}$

Based on this analysis of the Lewin Metal of Behavior, a behavioral factor system is established as follows. Eight kinds of main style factors are analyzed under the behavior impact factors system. Based on the evolution of the Lewin Metal of Behavior, the system is divided into three levels, including three categories and twelve sub-categories (As shown in Figure 10).

\subsubsection{Analytic Hierarchy Process on the Behavioral Factor System of the Main Factor in Diaolou}

Use AHP, combined with style system of Diaolou style building to explore the value of the weight of each behavioral factor. Proceed as follows:

1) establish a structural model of the hierarchical analysis (Figure 11);

2) construct a judgment matrix;

3) hierarchical single sorting;

4) consistency test;

5) hierarchical total sorting. Among them, the structural model is as follows.

The initial assignment of importance to the behavioral factor is based on the analysis of the actual usage of the main elements of the Diaolou style building in 6 typical Qiangzhai villages such as Taoping, Baishi, Pingtou, Hexin, Longxi and Xiouxi in Aba Prefecture. The initial assignment combines the questionnaire with experts' opinions.

Finally, weights of behavioral factors are respectively given weight coefficients according to the weight value of Diaolou-style factors, as shown in the following Table 2: 
To observe the weight of each behavioral factor visually, weight of the factor system is converted into the following graph (Figure 12):

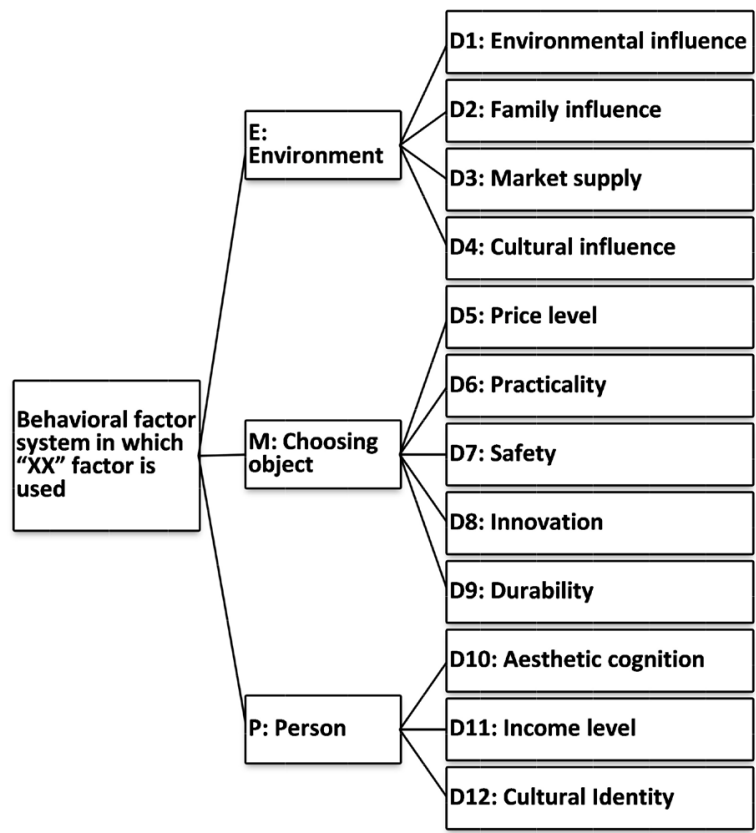

Figure 10. Behavioral factor system about the use of the style factors of Diaolou style building.

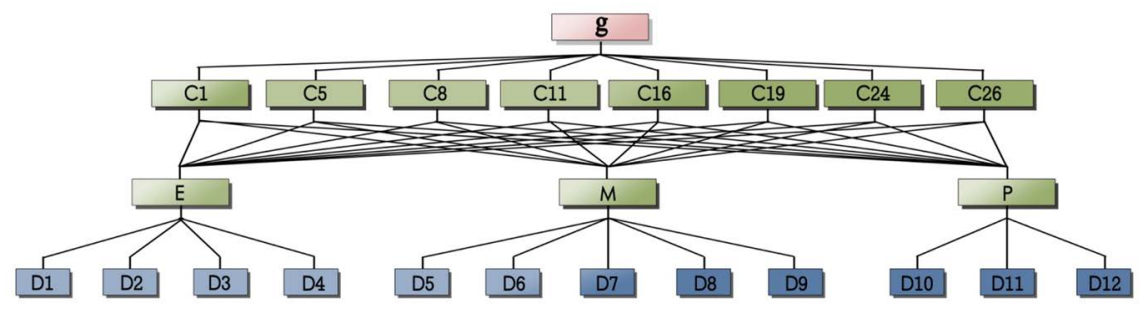

Figure 11. Analytical model of AHP.

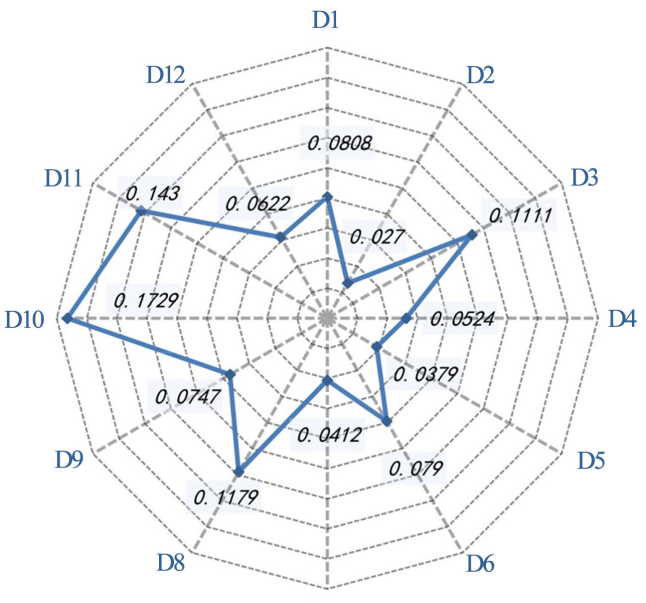

D7

$\multimap$ WeIGHT VALUE

Figure 12. The weight value of the factor affected by the behavior. 
Table 2. The value of the factor weight affected by the behavior.

\begin{tabular}{|c|c|c|}
\hline & $\begin{array}{l}\text { the use of the } \\
\text { architecture }\end{array}$ & Weight value about behavioral factors \\
\hline \multirow{4}{*}{$\mathrm{E}$} & D1 & 0.0808 \\
\hline & D2 & 0.027 \\
\hline & D3 & 0.1111 \\
\hline & D4 & 0.0524 \\
\hline \multirow{5}{*}{ M } & D5 & 0.0379 \\
\hline & D6 & 0.079 \\
\hline & D7 & 0.0412 \\
\hline & D8 & 0.1179 \\
\hline & D9 & 0.0747 \\
\hline \multirow{3}{*}{$\mathrm{P}$} & D10 & 0.1729 \\
\hline & D11 & 0.143 \\
\hline & D12 & 0.0622 \\
\hline
\end{tabular}

\subsubsection{Screening of Main Behavioral Impact Factors}

Through analyzing data, the behavioral impact factors are divided into three categories: $\geq 0.08,0.05-0.08, \leq 0.05$, which are defined as the main behavioral factors, secondary behavioral factors and general behavioral factors.

Among them, five kinds of behavioral impact factors such as aesthetic cognition, income, innovation, market supply and environment influence occupy a higher weight and are the main behavioral impact factors.

\section{Result}

\subsection{Control Strategy Based on Data Analysis}

Combined with style system of Diaolou style building, this paper puts forward suggestions that the expression of Diaolou style factor and the control of behavioral impact factor should be paid attention.

First of all, grasp the elements of the overall factors to create a Qiang Diaolou architectural style as a whole; grasp the architectural form, material, building entrance, window walls, railings, roofs, canopies and other places connected with style factors; discriminate the influences of person, choosing object and environment; grasp the overall style of style factor system of Diaolou style building.

Second, focus on the main factors in Diaolou architectural style factor system and the main factor in the behavioral impact factor system, especially the main factors of weight $\geq 0.05$ and main behavioral factors of weight $\geq 0.08$; grasp main factors from style characteristics and behavior control.

Third, in the process of building Diaolou architectural style, we should combine the practical operability, pay attention to the integrity of the expression of style factor to increase the correlation between the style model and the building entity, and pay attention to the implementation of the guidance control of the 
behavioral impact factor, so as to construct the Diaolou style building of Qiang people, which have both geographical features and cultural connotation.

\subsection{Behavior-Based Diaolou Architectural Style Guidance Strategy}

Diaolou architectural style control strategy should be based on data analysis; data analysis is served to make strategy accurate and oriented. Through the data, we should pay more attention to the impact factors of the five main types of behaviors in the actual construction. Due to the fact that style factors of Diaolou style building is restricted by various behavioral factors, five main types of behavioral factors should be guided and utilized appropriately in the use of Diaolou style factors. The specific analyses are as follows:

\subsubsection{Comprehensive Analysis of Behavioral Impact Factors}

Through an in-depth analysis of data, we can see that in the behavioral model $\mathrm{P}$ $=\{$ Aesthetic cognition, Income, Cultural Identity\}, aesthetic cognition and cultural Identity are more strongly affected by foreign cultures. Due to the remoteness and the low income level, the behavior of person tends to favor the foreign cultural value system.

In $\mathrm{E}=\{$ Family influence, Cultural influence, Environmental influence, Market supply\}, the original style features disappear in the market-oriented circulation on account of the cultural influence and the changes of the surrounding architectural environment, especially after getting rid of a relatively closed self-built model of the Qiang style buildings.

In $\mathrm{M}=\{$ Innovation, Practicality, Durability, Safety, Price level $\}$, the original style of the building is declining because of the disappearance of the functional features of the style factor and the problems of safety and durability.

\subsubsection{Diaolou Architectural Style Guidance Strategy Based on Behavior Analysis}

Fully exploring the data and analyzing predict-oriented change of style should be the premise of the guidance strategy of Diaolou style building of Qiang people. Combined with the research, data and equation $\mathrm{B}=f(\mathrm{P}, \mathrm{E}, \mathrm{M})$, it can be seen that the impact of foreign cultures makes the Qiangs question and even abandon their culture. This phenomenon gradually spreads from the field of thought to the production and life, affecting the Qiang Diaolou architectural style. Under this kind of impact, some factors that can be well adapted to the supply of the market have been developed and utilized, such as limestone group wall, etc., while other parts are gradually reduced, such as architecture climbing, semi-masking roof and semi-masking roof which are gradually transformed into a balcony, and the style features are gradually lost.

At the same time, due to the improvement of architectural skills and the renovation of building materials, the original functional architectural style factor gradually loses its function and can only exist in the form of decorative embellishment. Its existence value is transformed from original functionalism and 
formal aesthetics to single aesthetic value. Typical examples are body contraction and limestone group wall, which only serve as the decoration of the outer skin of the building. However, the actual use of such factors is gradually decreasing since the aesthetic cognition is influenced by foreign cultures.

Through analysis, it is necessary to change the status of the Diaolou style building of Qiang people that style factors are gradually abandoned, so that they can be fully utilized. This article makes the following suggestions:

First, in order to protect ethnic style and traditional culture, we should attach importance to style factors of main Diaolou style building of Qiang people and combine with the analysis of style factors. A better style shaping effect can be achieved if the application of the main style factors is emphasized from design to construction.

Second, start from the impact factors of usage behavior that influence Diaolou architectural style and achieve major breakthroughs. Make a fully understanding of style factors and behavioral factors to make a targeted step. Therefore, style factors are closer to Diaolou architectural style factor system.

Third, explore the intrinsic meaning of the data, and turn transfusion-like style protection into a hematopoietic one. Make a transformation of style protection from being affected by foreign culture to be widely used in the cultural communication and diffusion. The following points are put forward.

Firstly, building Diaolou architectural aesthetics based on the Diaolou architectural style factor. Strive to find ways to let Diaolou style building of Qiang people make an influence on modern buildings, halls, hotels and houses. Let Diaolou style building play an important role in unique aesthetics.

Secondly, we need to make an improvement and innovation of Diaolou style factors. The existing Diaolou style factors gradually lose their functional significance, and generally exist as aesthetic components of the national emblems. Such factors need to be improved in conjunction with the use of modern buildings to make them not only aesthetic but also practical.

Thirdly, strengthen the combination of Diaolou style factors and the current architectural style. Combine Diaolou with existing buildings in terms of color, form, function and use, especially with modern high-rise buildings to make Diaolou style building of Qiang people blend into the current and mainstream architecture.

\section{Discussion}

1) Research methods and mechanisms: this research method is not only a combination of Analytic Hierarchy Process and Lewin Metal of Behavior, but a combination of qualitative and quantitative analysis. At the same time, it analyzes the use of the style factors in the Qiang region objectively and quantitatively, and deconstructs the weight of each factor by data.

2) Data analysis: the selection of the initial data of this study is based on the full cognition of current situation. Since the main problem of this study is to 
quantitatively describe the subjective problems objectively, subjective data is chosen as the initial data.

First, in order to select Qiang style factors, initial style factors and main impact factors in the Metal of Behavior, current situation needs to be taken into account.

Second, the initial data of Analytic Hierarchy Process is still subjective to a certain extent. Experts in charge of consulting of Analytic Hierarchy Process include senior designers of Architectural Design Institute, researchers of University School of Architecture, graduate students majored in architecture and other professionals. The questionnaire adopts a nine-level evaluation system to analyze subjective issues objectively as much as possible.

3) Result analysis: according to the Analytic Hierarchy Process and the Lewin Metal of Behavior, there are eight main types of style factors: limestone group wall, wooden lattice window, white stone decorated roof, white stone eave, ethnic minority window covering, Nase, body contraction and sheep totem. Based on this analysis, the main behavioral factors are analyzed, including five impact factors: aesthetic cognition, income level, innovation, market supply and environment influence.

The research process data meets the requirements of the credibility of AHP, and conforms to the actual construction of 6 typical Qiang villages including Taoping, Baishi, Pingtou, Hexin, Longxi and Xiouxiin Aba Prefecture.

The results of the analysis can not only guide the construction of Qiang architectural style, but also provide a theoretical reference for the directional policy of government-defined protection.

4) Innovation and limitations: research methods and research perspectives are innovative, but there are limitations in the research process.

Innovation of the Method: the innovation of this research lies in the perpetuation of subjective and behavioral problems.

Innovation of research perspective: Diaolou architectural style is analyzed from the perspective of behavior.

Methodological limitations: there is some subjectivity in the initial assignment.

Limitations of research perspective: first, it fails to combine the strategy of style protection and the production and development of Qiang ethnicity from the perspective of economic development. Second, it fails to refine and improve the control and guidance strategy of usage behavior from the perspective of development of local productive force.

\section{Conclusions}

From behavioral perspective, this paper innovatively uses the Lewin metal of behavior and Analytic Hierarchy Process to study the style system and behavioral factor system of Diaolou style building of Qiang people, and puts forward a control and guidance strategy of inheritance and development of Qiang Diaolou 
architectural style.

On this basis, this paper proposes that we should master the influencing factors as a whole, and control and guide those whose weight is greater than 0.05 at first.

Second, in the expression of factors, we should pay attention to the integrity of the expression of style factor and the implementation of the guidance control of the behavioral impact factor, and follow the principle of people-oriented, compound function, dynamic development and highlighting national aesthetics. So that the architectural style system of Diaolou style building of Qiang people can be developed continuously.

Third, in the protection of traditional ethnic styles, we should focus on style factors in the first instance. We should take a fully understanding of style factors and behavioral factors in order to make a targeted step. Therefore, style factors are closer to Diaolou architectural style factor system. In the end, we should pay attention to the application of the main style factors during the integration process from design to construction, so that it achieves a better style shaping effect.

Fourth, we should build Diaolou architectural aesthetics and combine with the use of modern buildings to make an improvement and innovation of Diaolou style factors. Combine Diaolou with existing buildings in terms of color, form, function and usage.

Fifth, the development of Diaolou style building of Qiang people should be based on its style system, and grasp the trend of architectural style. At the same time, on the basis of usage behavior impact system of style factor, it implements usage behavioral guidance strategy and theory of development, providing a theoretical and technical guidance for the heritage of Qiang Diaolou architectural style and the government's directional policy.

In the future, we hope to incorporate more discipline ideas into the study of regional architectural styles, such as architecture, humanities, psychology, etc. We can also innovate to adopt more research methods. In addition, we hold that it should be raised to urban perspective on the study of regional architectural style. The work of this paper is only a preliminary test on the factor elements of Qiang Diaolou architectural style. We can also introduce the ideas and methods of morphological typology, and carry out in-depth exploring of style elements, combination rules and technical methods for the derivation of various elements in the future. Furthermore, it will be upgraded to the level of form design. By establishing databases of individual buildings and streetscape, etc., the research results of style factors can be better utilized in the whole process of urban planning and construction. Finally, we should deepen the connection between the technical level and the various levels of planning, and clearly define the content composition, operational rules and depth requirements of the urban design guidelines, making it the form-referenced, database-based design schema language for review and approval of planning and architectural design. It provides necessary theoretical and technical guidance for local governments and planners. 


\section{Acknowledgements}

From the beginning of the investigation to the completion of writing, this study lasted nearly three months. I am particularly grateful for those experts and researchers who are involved in the research;

At the same time, I would like to express my gratitude to all the scholars who wrote these theses. This study would not come into being without the help and inspiration from the theses of these scholars.

\section{Funding}

The work was supported by the fund of the Science and Technology Department of Sichuan Province. The project name: Innovative design study of the Qiang stone masonry building (Grant NO: 18ZDYF3350).

\section{Conflicts of Interest}

The authors declare no conflicts of interest regarding the publication of this paper.

\section{References}

[1] Ma, N. and Qian, Y. (2006) Analyzing the Bunker's Construction and Culture of Qiang Nationality. Journal of Xihua University. Philosophy \& Social Sciences, No. 03, 20-23.

[2] Li, X., Zeng, Y. and Ji, F. (2001) The Interrelation between Primitive and Modern Conception of the Civilian Blockhouses in Qiang Nationality. Journal of Sichuan University of Science and Technology, No. 02, 46-49.

[3] Yu, Z. (2006) Study of the Traditional Housing Eco-Design of Diao Lou of the Qiang Minority. Journal of Fujian University of Technology, No. 03, 310-312.

[4] Yong, C. (2009) On the Earthquake Resistance of Watchtowers after Wenchuan Earthquake. Journal of Southwest University (Natural Science Edition), No. 05, 1077-1080.

[5] Zhang, H. (2008) Research on the Culture and Civil Dwelling Houses of the Qiang Minority. Journal of Chengdu University (Social Sciences), No. 02, 60-65.

[6] Ji, F. (2008) Exploration of Folklore of Sichuan Human Settlements. South Architecture, No. $05,5-10+14$.

[7] Ji, F. (2010) The Initial Research of Houses and Settlements of the Three. Chongqing Architecture, No. 12, 1-3.

[8] Gan, L. (2016). Study on Spatial Cultural Architectural Elements of Qiang Ethnic minority. Shanxi Architecture, No. 23, 24-25.

[9] Peng, D. and Tang, G. (2007) The Factor for Diao Lou Beauty of Qiang People. Journal of Southwest University for Nationalities (Humanities and Social Science), No. 06, 51-53.

[10] Yang, B., Fan, Z. and Guo, M. (2009) Rescuing and Protective Rehabilitation for the Folk's House of Qiang Nationality in Luobozhai, Wenchuan after the Disaster. Archicreation, No. 12, 58-65.

[11] Wan, J., Zhang, Y. and Li, B. (2011) On Historic Qiang Autonomous Style Buildings. Chongqing Architecture, No. 12, 43-45. 
[12] Ren, H. (2003) The Building and Stockaded Village of Qiang Nationality. Architectural Journal, No. 08, 62-64.

[13] $\mathrm{Wu}, \mathrm{Q}$. and $\mathrm{Hu}, \mathrm{Q}$. (2015) The Simple Analysis of the White Stone's Semiology for the Qiang People's Dwellings. Architecture \& Culture, No. 06, 184-185.

[14] Lu, W., Wang, J. and Jiang, L. (2011) Deficiency and Reconstruction of Qiang Traditional Architectural Aesthetic Values in Restoring Beichuan. Journal of Northwestern Polytechnical University (Social Sciences), No. 01, 52-54.

[15] Liu, W. and Liu, B. (2011) An Analysis of the Space Design Culture of Qiang Village-Housing. Journal of Qingdao University of Technology, No. 03, 58-62.

[16] Yuan, L. (2006) Research of Buildings Distribution and Building Characteristic about a Ruins of Ancient Village. Sichuan Building Science, No. 6, 205-209.

[17] Han, Q. (2010) Beichuan Qiang Memory and Architecture Localization. Development of Small Cities \& Towns, No. 3, 78-80.

[18] Zhao, X. and Liu, M. (2010) Guardianship and Identification: Cultural Significance of the Dialogue between Lese Building of Qiang Minority and the Han Religious Building. Northwestern Journal of Ethnology, No. 2, 204-209+136.

[19] Hao, Z. (2010) Explored Architectural Expression of Qiang Nationality Culture Based on Post-Disaster Reconstruction. Sichuan Building Science, No. 3, 280-282.

[20] Zhao, X. and Wu, T. (2010) On the Regeneration and Sustainable Growth of Ethnic Culture in Post-Quake Periods-A Case Study of the Architectural Culture and Intangible Cultural Reconstruction in the Village of Xiuxi of the County of Lixian. Journal of Aba Teachers College, No. 2, 18-23.

[21] Saaty, T.L. (2006) Rank from Comparisons and from Ratings in the Analytic Hierarchy/Network Processes. European Journal of Operational Research, No. 168, 557-570. https://doi.org/10.1016/j.ejor.2004.04.032

[22] Saaty, T.L. (2008) Decision Making with the Analytic Hierarchy Process. International Journal of Services Sciences, 1, 83-98. https://doi.org/10.1504/IJSSCI.2008.017590

[23] Lewin, K. (1951) Field Theory in Social Science. China Media University Press, Beijing.

[24] Jencks, C. (2002) The New Paradigm in Architecture: The Language of Post-Modernism. Yale University Press, London.

[25] Lynch, K. (1960) The Image of the City. Massachusetts Institute of Technology, Cambridge and London, The MIT Press, Cambridge.

[26] Xie, H. and Liu, L. (2003) Research Advance and Index System of Rural Landscape Evaluation. Chinese Journal of Ecology, No. 6, 97-101.

[27] Yang, Z. (2009) Research and Evaluation of Rural Settlements Landscape in Shanghai. Shanghai Jiaotong University, Shanghai.

[28] Che, S., Yang, Z. and Ni, X. (2008) Investigation of Landscape Patterns and Study on Landscape Element Design Patterns of Shanghai Countryside. Chinese Landscape Architecture, No. 8, 21-27.

[29] Yang, Z. and Che, S. (2010) Research on the Landscape of Shanghai Rural Settlements. Journal of Shanghai Jiaotong University (Agricultural Science), No. 3, 225-231. 